\title{
Electron Tomography of Crystalline Microstructures by Diffraction Contrast Imaging: Application to Ordered Alloys
}

\author{
S. Hata, ${ }^{*}$ K. Kimura, ${ }^{*}$ T. Horiuchi, ${ }^{* *}$ S. Matsumura, ${ }^{* *}$ and Y. Tomokiyo* \\ * Dept. of Appl. Science for Electronics and Materials, Kyushu Univ., Kasuga 816-8580, Japan \\ ** Dept. of Appl. Quantum Physics and Nuclear Engng., Kyushu Univ., Fukuoka 812-8581, Japan
}

For electron tomography of crystalline materials, diffraction effects are considered undesirable since the image contrast is sensitive to the direction of the incident electron beam as well as the thickness and density of the specimen [1-3]. On the other hand, diffraction contrast images can reveal various kinds of crystalline microstructures, such as lattice defects, grain boundaries, orientation variants of ordered domains, etc. Here we show an application of electron tomography using the diffraction contrast imaging to ordered alloys. Obtained results demonstrate that the diffraction contrast imaging can be utilized in the electron tomography.

The specimen is of a $\mathrm{Ni}-\mathrm{Mo}$ alloy $\left(18\right.$ at.\% Mo) in which $D 1_{\mathrm{a}}$-ordered $\mathrm{Ni}_{4} \mathrm{Mo}$ precipitates are formed in the Ni matrix [4]. When the tetragonal $\mathrm{Ni}_{4} \mathrm{Mo}$ precipitates are formed in the face-centered cubic (fcc) Ni matrix, six types of orientation variants can exist. Dark-field TEM (DF-TEM) can image a single $D 1_{\mathrm{a}}$ variant by selecting its superlattice reflection for the imaging [5]. The specimen was placed on the specimen holder where the $[420]_{\mathrm{fcc}}$ direction in the observation area is parallel to the tilt axis of the holder and the $D 1_{\mathrm{a}}$ superlattice reflection, indicated by the arrows in Fig. 1, is excited up to $\pm 60^{\circ}$ of the tilt angle.

For obtaining a tilt series of DF-TEM images, careful setting of the diffraction condition is necessary to reduce changes in the image contrast with tilting the specimen. When a deviation from the exact Bragg condition is significant or non-systematic reflections are strongly excited, a hollow or contour contrast appears in the precipitates, as shown in Figs. 2(a) and (b), respectively. Such undesirable diffraction contrasts can be reduced by changing the direction of the incident electron beam using a beam-tilt function, as shown in Figs. 2(c) and (d). Figure 3 shows part of the resultant tilt series of DF-TEM images of the $\mathrm{Ni}_{4} \mathrm{Mo}$ precipitates. The hollow and contour contrasts in the precipitates are mostly eliminated. The tomographic reconstruction in Fig. 4 obtained from this tilt series visualizes three-dimensional (3D) shapes and distribution of the $\mathrm{Ni}_{4} \mathrm{Mo}$ precipitates with a single $D 1_{\mathrm{a}}$ variant [4]. This diffraction contrast imaging technique will expand the applicability of electron tomography to 3D microstructure analysis of crystalline materials [6].

[1] M. Koguchi et al., J. Electron Microsc. 50 (2001) 235.

[2] P. A. Midgley and M. Weyland, Ultramicrosc. 96 (2003) 413.

[3] G. Möbus, R. C. Doole and B. J. Inkson, Ultramicrosc. 96 (2003) 433.

[4] L. A. Nesbit and D. E. Laughlin, Acta Metall. 26 (1978) 815.

[5] E. Ruedl, P. Delavignette and S. Amelinckx, Phys. Stat. Sol. 28 (1968) 305.

[6] K. Kimura et al., in this volume.

[7] This work was supported in part by the Grant-in-Aid for Scientific Research B from the Japan Society for the Promotion of Science, Grant-in-Aid for Exploratory Research from the Ministry of Education, Culture, Sports, Science and Technology and a grant from the Interdisciplinary Graduate School of Engineering Sciences, Kyushu University, Japan. 

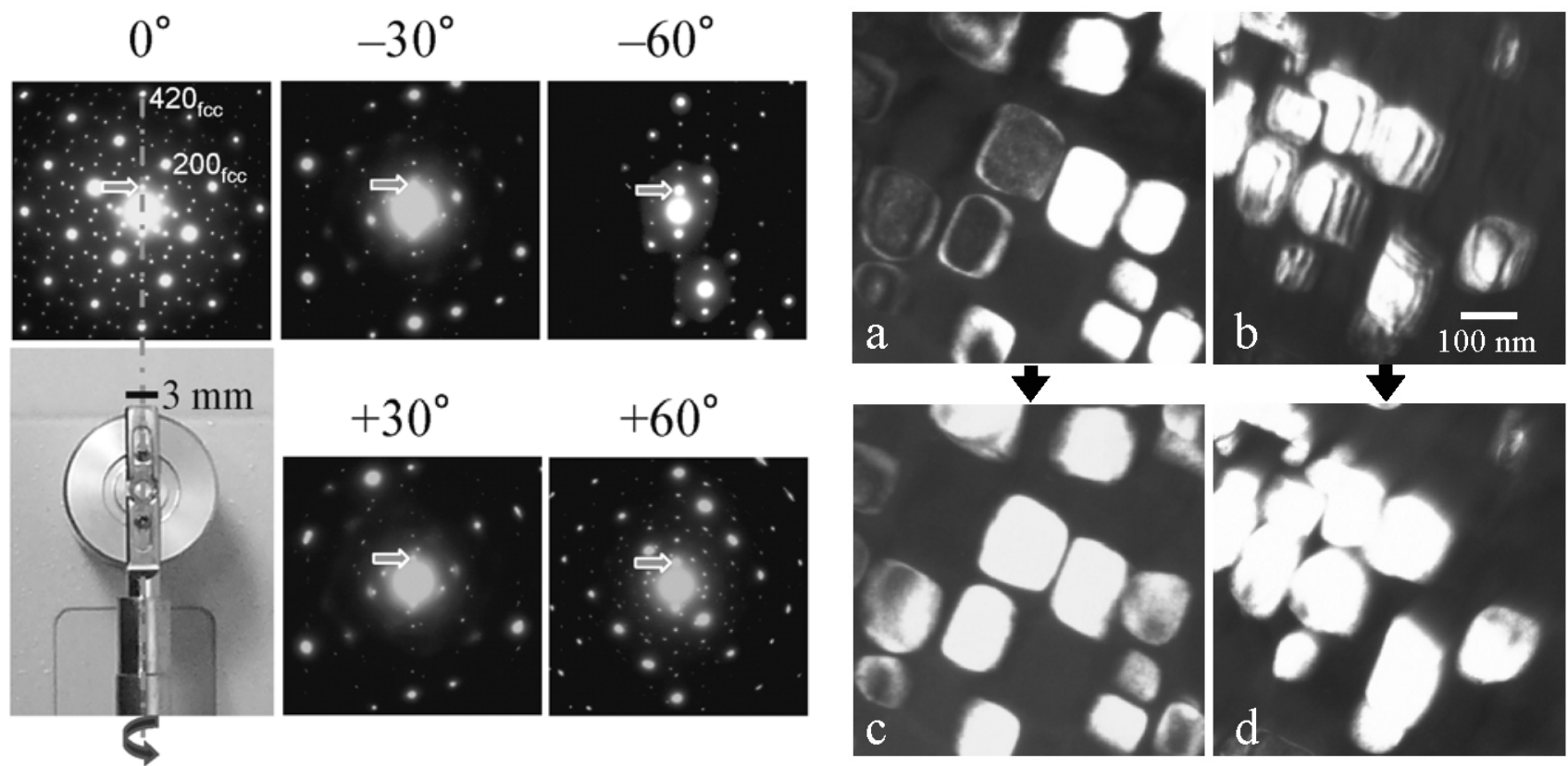

Fig. 1(Left). Part of a tilt series of electron diffraction patterns in a $D 1_{\mathrm{a}}$-ordered Ni-Mo alloy and a photograph of the specimen holder. Arrows indicate the $D 1_{\mathrm{a}}$ superlattice reflection used for imaging.

Fig. 2(Right). DF-TEM images of the $\mathrm{Ni}_{4} \mathrm{Mo}$ precipitates before (a, b) and after (c, d) improving the diffraction condition by tilting the incident electron beam.
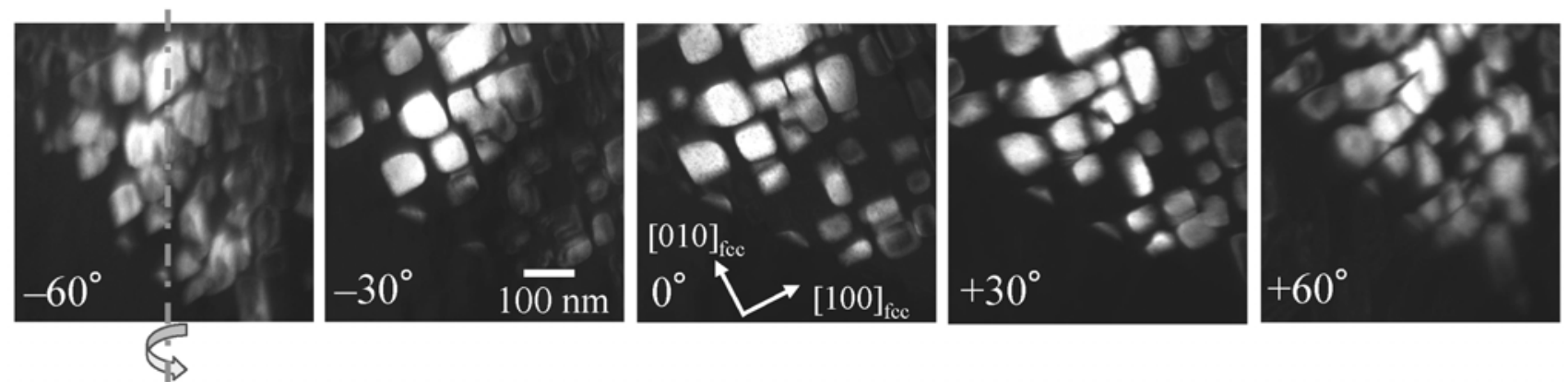

Fig. 3. Part of a tilt series of DF-TEM images of the $\mathrm{Ni}_{4} \mathrm{Mo}$ precipitates with one $D 1_{\mathrm{a}}$ variant.

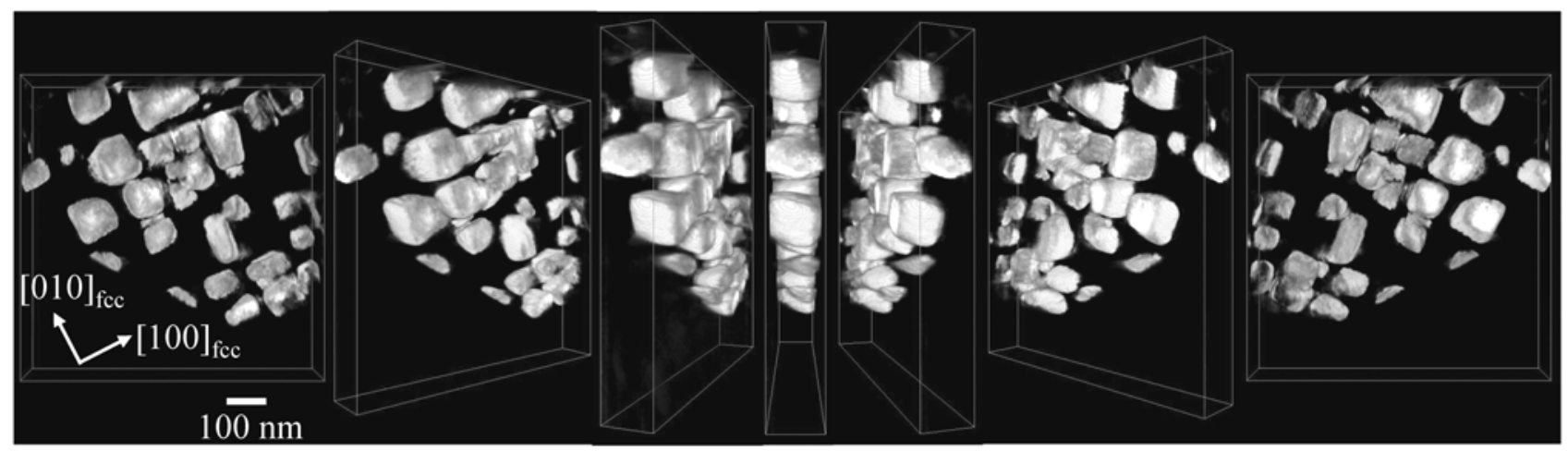

Fig. 4. Three-dimensional views of the $\mathrm{Ni}_{4} \mathrm{Mo}$ precipitates reconstructed from the tilt series in Fig. 3. 